\title{
MARGINAL CHIPPING OF THREE DIFFERENT CAD/CAM CERAMIC VENEERING MATERIALS AS INFLUENCED BY MACHINABILITY
}

\author{
Carl Hany* and Maha Tymour*
}

\begin{abstract}
Statement of the problem: The curiosity of many prosthodontists was attracted towards CAD/ CAM technology combined with the newly specifically designed ceramic materials to achieve superb esthetic restorations for their patients. The new ceramic materials with their different mechanical properties might have an impact on their machinability particularly for producing thin esthetic restorations.
\end{abstract}

Purpose: The purpose of this investigation was to evaluate the marginal quality of three different ceramic veneers: Leucite reinforced, hybrid and lithium di-silicate in terms of edge chipping and chipping factor using different machining protocols.

Materials and methods: In order to achieve the purpose of this study, a maxillary central incisor of a Typodont was prepared for a feather edge veneer preparation to receive forty five ceramic veneers that were milled from three different materials using the Cerec CAD/CAM system. The three tested materials were leucite-reinforced glass ceramic (IPS Empress CAD), hybrid ceramic (Vita Enamic), and lithium di-silicate glass ceramic (IPS e.max CAD). The forty five veneers were classified into three groups according to the type of the material fifteen each, that were further divided into three subgroups of five specimens each according to the three tested machining speeds: normal, fast, and two-step. A digital camera was used for taking top images for the tested veneer margins that were imported into image analysis software to measure the peripheral veneer circumference $(\mathrm{P})$. A stereomicroscope was used to measure the amount of marginal chipping of each veneer in microns $(\mathrm{L})$. The chipping factor $(\mathrm{CF})$ was calculated using the following equation: $\mathrm{CF}=\mathrm{L} / \mathrm{P} \times 100$, where $(\mathrm{L})$ is the amount of marginal chipping and $(\mathrm{P})$ is the marginal circumference of each veneer. The data of the chipping factor of each veneer margins were recorded and tabulated to be statistically analyzed in order to detect the influence of either the ceramic material or the machining protocol on the quality of the veneer margins in terms of edge chipping.

Results: Effect of ceramic type regardless of machining protocol: The statistical analysis of the obtained data revealed that e.max CAD (LD) showed the statistically significant highest mean chipping factor value ( $7.25 \pm 2.56$ ) followed by IPS Empress CAD (LR) (4.01 \pm 0.83 ), and the least mean value was obtained with Vita Enamic (HC) $(2.83 \pm 1.56)$.

\footnotetext{
* Associate Professor, Fixed Prosthodontics Department, Faculty of Dentistry, Cairo University
} 
Effect of machining protocol regardless of ceramic type: there was a statistically significant difference between the three milling techniques, where fast milling showed the highest mean chipping factor value $(6.30 \pm 2.97)$, followed by normal milling $(4.89 \pm 2.22)$ where the least chipping factor mean value $(2.90 \pm 1.60)$ was obtained with the two-step milling.

\section{Conclusions:}

1- The edge quality of thin ceramic CAD/CAM restorations proved to be material dependent.

2- The machinability for producing thin $\mathrm{CAD} / \mathrm{CAM}$ ceramic restorations influenced significantly their marginal quality.

3- The prosthodontist should be aware and knowledgeable about the mechanical properties of the recent $\mathrm{CAD} / \mathrm{CAM}$ ceramic materials in order to choose the optimum one with the convenient milling protocol during the treatment planning to get the best clinical performance.

\section{INTRODUCTION}

The continuing progress in CAD/CAM technology provided the fixed prosthodontist with restorations having good marginal quality comparable to that produced with laboratory fabrication. ${ }^{1}$ The application of minimum intervention procedures is considered one of the main advantages of $\mathrm{CAD} /$ CAM technology. ${ }^{2}$ Based on the current evidence from the dental literature, the application of minimally invasive preparation designs using the CAD/ CAM technology was found to be a successful treatment option. ${ }^{3-6}$ This was proved to be true particularly with thin labial and occlusal ceramic veneers. ${ }^{6,7-9}$ The marginal edges of the ceramic restorations are very critical areas that play a very determinant role in the success of this type of esthetic restorations. In addition to accuracy of fit of these margins either vertically or horizontally; the edge quality as expressed by its smoothness and being free of discrepancies and irregularities in the form of chipping fractures are reported to substantially influence clinical lifetimes. ${ }^{10-12}$ Small chipping fractures of the restoration margins were found to be responsible for late clinical failures of ceramic restorations. ${ }^{12-14}$ These marginal chippings are in fact patent defects that would be initially covered by the luting composite, inducing small crack growth and reducing the clinical success rate. The marginal discrepancies in addition would expose the luting cement to the oral environment, leading to a more aggressive rate of cement dissolution by the oral fluids and chemomechanical forces. This might compromise the longevity of the tooth by caries and periodontitis. ${ }^{15,16}$ As the CAD/CAM technology is continually subjected to enormous modifications and innovations; new esthetic dental ceramics and polymer-based materials are also introduced with desirable properties aiming for the best functional and esthetic performance.${ }^{5,6,17-20}$ Rekow, ${ }^{21}$ reported that there is a potential for generation of machining induced damage through utilizing abrasive tools of the $\mathrm{CAD} / \mathrm{CAM}$ systems for either grinding or milling that could reduce the integrity of the final restoration. Therefore, the machinability of the chosen ceramic material could affect the integrity of the minimally invasive restorations like thin veneers. Shearer et al, ${ }^{22}$ and Sindel et al, ${ }^{23}$ found that the surface damage of ceramic materials due to machining was observed as chipping defects that can reduce the accuracy of fit and contribute to reduction of the restoration strength. Tsitrou et al, ${ }^{24}$ described the machinability as the ease with which a given material is cut. Baik et al, ${ }^{25}$ Taira and Yamaki, ${ }^{26}$ suggested various parameters for quantitative assessment of machinability, such as tool wear, surface roughness, cutting force and drilling rates. On the other hand, Boccaccini, ${ }^{27}$ proposed another method for estimating the material machinability through quantifying its brittleness. 
Sehgal et al, ${ }^{28}$ and Boccaccin, ${ }^{29}$ determined the brittleness of glass-ceramics and glass matrix composites by indentation data.

Tsitrou et $\mathrm{al}^{24}$ in their research estimated the degree of marginal chipping of different machinable esthetic materials using the CEREC system through introducing the chipping factor (CF) as a new concept. They calculated the chipping factor by estimating the ratio of overall marginal chipping over the total marginal circumference of the restoration multiplied by 100 to give the percentage of chipping. Porto et al, ${ }^{30}$ reported that the marginal chipping factor (CF) can be calculated only after these esthetic restorations have been milled. They also reported that ceramics have a higher brittleness index when compared with other hybrid or composite materials.

Furthermore, Chavali et al, ${ }^{31}$ studied in vitro the tool penetration rate for two polymer-containing CAD-CAM materials (Lava Ultimate and Enamic) and two ceramic-based CAD-CAM materials (e.max CAD and Celtra Duo). They found that Lava Ultimate and Enamic have greater machinability and less edge chipping than e.max CAD and Celtra Duo. Therefore, they ${ }^{30,31}$ reported that CAD/CAM polymer-based esthetic materials might have a better clinical performance than glass-ceramics.

There is still little information available in the dental literature regarding the machinability of the new CAD/CAM esthetic materials that are particularly used for thin veneers. Therefore, the main interest of the present research was mainly directed toward assessment of the marginal quality of three different esthetic materials used for anterior veneers in terms of edge chipping when subjected to three different milling speeds.

The null hypothesis of the present investigation was that there would be no significant difference concerning the restoration edge chipping irrespective of the material type or the machining speed program.

\section{MATERIALS AND METHODS}

Forty-five anterior laminate veneers were milled from three different ceramic materials using $\mathrm{CAD} /$ CAM milling unit (CEREC MC XL Sirona, Dental, Bensheim, Germany). The veneers were produced from a feather edge veneer preparation design of a maxillary central incisor acrylic tooth of a Typodont model(NISSIN Dental Model, Koyoto, Japan). ${ }^{32}$ The three materials selected for the present research were leucite-reinforced glass ceramic (LR) IPS Empress CAD (Ivoclar Vivadent, Schaan Liechtenstein), Vita Enamic Hybrid Ceramic (HC) (Vita Zahnfabric), and lithium disilicate glass ceramic (LD) IPS e.max CAD (Ivoclar Vivadent, Schaan Liechtenstein). The forty five ceramic laminates were constructed following the standard three phases of CAD/CAM process, ${ }^{32}$ and were classified to three groups of fifteen specimen per each. For the milling process of each material, a new set of burs was used. Each group was divided into three subgroups of five specimens for each (Table 1) according to the three tested milling speeds: Normal (8.35 mins), Fast (5.08 mins) and Two-step (15.57 mins) in order to test this speed machining programs on the marginal chipping of the three different ceramic materials.

TABLE (1) Ceramic veneers distribution according to material type and milling speed.

\begin{tabular}{|c|c|c|c|c|}
\hline \multirow{2}{*}{ Ceramic Materials } & \multicolumn{3}{|c|}{ Milling Speed } & \multirow{2}{*}{ Total } \\
\cline { 2 - 4 } & Normal & Fast & Two-step & \\
\hline $\begin{array}{c}\text { IPS Empress CAD } \\
\text { Leucite reinforced (LR) }\end{array}$ & 5 & 5 & 5 & 15 \\
\hline $\begin{array}{c}\text { Vita Enamic Hybrid } \\
\text { Ceramic (HC) }\end{array}$ & 5 & 5 & 5 & 15 \\
\hline $\begin{array}{c}\text { IPS e.max CAD } \\
\text { Lithium disilicate (LD) }\end{array}$ & 5 & 5 & 5 & 15 \\
\hline Total & 15 & 15 & 15 & 45 \\
\hline
\end{tabular}

The quantitative analysis of the marginal chipping was done as described before in two previous studies by Gianntopoulos et al, ${ }^{18}$ and Tsitrou et al, ${ }^{24}$ through calculating the chipping factor (CF) 
of each restoration using the following equation $\mathrm{CF}=\mathrm{L} / \mathrm{P} \times 100$ where $\mathrm{L}$ is the amount of marginal chipping and $\mathrm{P}$ is the marginal circumference of the restoration multiplied by 100 to give the percentage of chipping.

A top view image of the margins of each veneer (Fig. 1) was taken using a digital camera (Kodak/ Nikon DCS 410) connected to a PC (MacBook Pro, Intel core i5) to measure the peripheral circumference $(\mathrm{P})$ of the veneer using the Adobe Photoshop software (Adobe Inc. system V5. 0. Ltd. Europe). The images were then imported into Image Pro Plus software (V4. 01, Media Cybernetics, USA). The average periphery of all the veneer margins was calculated and found to be $33.0 \pm 0.01 \mathrm{~mm}$. Analysis of the marginal quality as well of each veneer from the different aspects: cervical, axial, and incisal through distribution of the chipping defects was performed. The length of the chipped margins of each veneer was measured using a stereomicroscope (Leica, Germany), at magnification $25 \mathrm{X}$ and the total amount of each specimen was calculated in microns (L). The chipping factor $(\mathrm{CF})$ of each ceramic veneer in the different experimental groups was recorded and tabulated to be statistically analyzed in order to detect the effect of the different tested machining protocols and the ceramic materials, as well, on the edge chipping of the ceramic veneers.

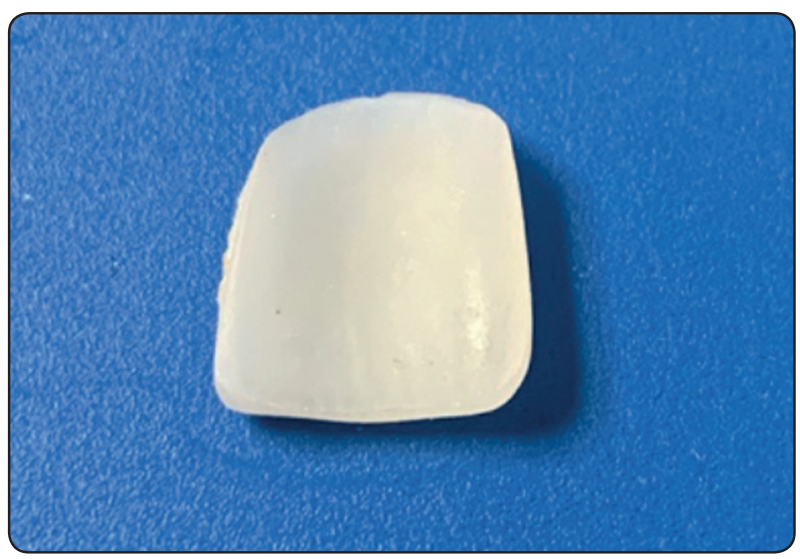

Fig. (1) Top view image showing the peripheral margins of one of the tested e.max CAD ceramic veneers.
In order to ensure the accuracy of the results, all the measurements were taken by the two authors separately in different times and correlated, and were found to be nearly identical. The mean of the two readings was calculated to be the actual written data.

\section{RESULTS}

Numerical data were explored for normality by checking the distribution of data and using tests of normality (Kolmogorov-Smirnov and ShapiroWilk tests). Data showed normal (parametric) distribution. Data were presented as mean and standard deviation (SD) values. Two-way Analysis of Variance (ANOVA) was used to study the effect of ceramic type, milling and their interaction on mean chipping factor. Bonferroni's post-hoc test was used for pair-wise comparisons when ANOVA test is significant. The significance level was set at $\mathrm{P} \leq 0.05$. Statistical analysis was performed with IBM SPSS Statistics for Windows, Version 23.0. Armonk, NY: IBM Corp.

\section{Two-way ANOVA results}

The results showed that ceramic type regardless of milling had a statistically significant effect on mean chipping factor. Milling regardless of ceramic type had a statistically significant effect on mean chipping factor. The interaction between the variables also had a statistically significant effect on mean chipping factor. Since the interaction between the variables is statistically significant, so the variables are dependent upon each other (Table 2).

\section{Effect of ceramic type regardless of milling protocol}

Regardless of milling; there was a statistically significant difference between mean chipping factor of the three ceramic types ( $P$-value $<0.001$, Effect size $=0.969)$. Pair-wise comparisons revealed that e.max showed the statistically significantly highest mean chipping factor value. IPS Empress CAD showed statistically significantly lower mean value. 
TABLE (2) Two-way ANOVA results for the effect of different variables on mean chipping factor

\begin{tabular}{lcccccc}
\hline \multicolumn{1}{c}{ Source of variation } & $\begin{array}{c}\text { Type III Sum } \\
\text { of Squares }\end{array}$ & df & $\begin{array}{c}\text { Mean } \\
\text { Square }\end{array}$ & F-value & P-value & $\begin{array}{c}\text { Effect size (Partial } \\
\text { eta squared) }\end{array}$ \\
\hline Ceramic type & 156.894 & 2 & 78.447 & 557.796 & $<0.001^{*}$ & 0.969 \\
Milling & 87.902 & 2 & 43.951 & 312.512 & $<0.001^{*}$ & 0.946 \\
Ceramic type x Milling interaction & 42.432 & 4 & 10.608 & 75.428 & $<0.001^{*}$ & 0.893 \\
\hline
\end{tabular}

$d f:$ degrees of freedom $=(n-1), *:$ Significant at $P \leq 0.05$

TABLE (3) The mean, standard deviation (SD) values and results of two-way ANOVA test for comparison between chipping factor of ceramic types regardless of milling protocol

\begin{tabular}{|c|c|c|c|c|c|c|c|}
\hline \multicolumn{2}{|c|}{ IPS Empress CAD } & \multicolumn{2}{c|}{ Vita Enamic } & \multicolumn{2}{c|}{ e.max } & \multirow{2}{*}{ P-value } & $\begin{array}{c}\text { Effect size (Partial } \\
\text { eta squared) }\end{array}$ \\
\cline { 1 - 5 } Mean & SD & Mean & SD & Mean & SD & & 0.969 \\
\hline
\end{tabular}

*: Significant at $P \leq 0.05$, Different superscripts are statistically significantly different

TABLE (4) The mean, standard deviation (SD) values and results of two-way ANOVA test for comparison between chipping factor values with different milling techniques regardless of ceramic type

\begin{tabular}{|c|c|c|c|c|c|c|c|}
\hline \multicolumn{2}{|c|}{ Normal milling } & \multicolumn{2}{c|}{ Fast milling } & \multicolumn{2}{c|}{ Two-step milling } & \multirow{2}{*}{ P-value } & $\begin{array}{c}\text { Effect size (Partial eta } \\
\text { squared) }\end{array}$ \\
\cline { 1 - 5 } Mean & SD & Mean & SD & Mean & SD & & 0.946 \\
\hline
\end{tabular}

*: Significant at $P \leq 0.05$, Different superscripts are statistically significantly different

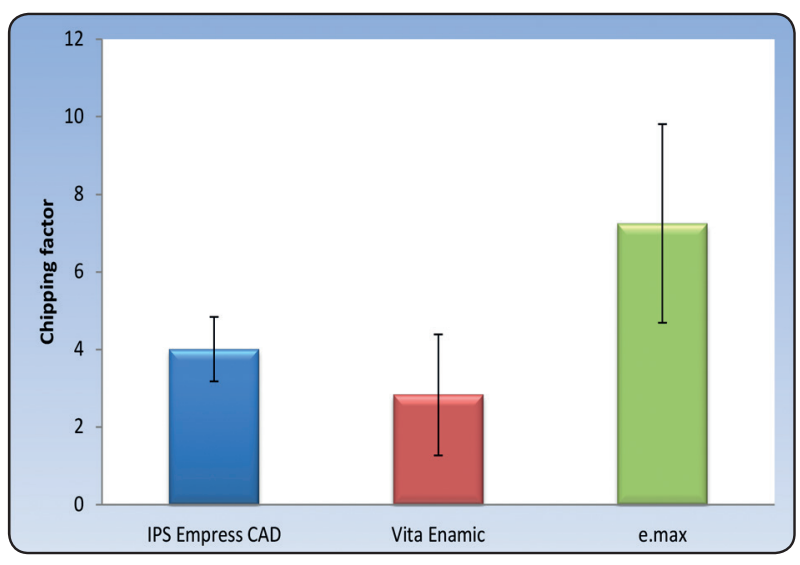

Fig. (2). Bar chart representing mean and standard deviation values for chipping factor values of ceramic types regardless of milling protocol.
Vita Enamic showed the statistically significantly lowest mean chipping factor value (Table 3, Fig 2).

\section{Effect of milling regardless of ceramic type}

Regardless of ceramic type; there was a statistically significant difference between mean chipping factor of the three milling techniques $(P$-value $<0.001$, Effect size $=0.946)$. Pair-wise comparisons revealed that fast milling showed the statistically significantly highest mean chipping factor value. Normal milling showed statistically significantly lower mean value. Two-step milling showed the statistically significantly lowest mean chipping factor value (Table 4, Fig 3). 


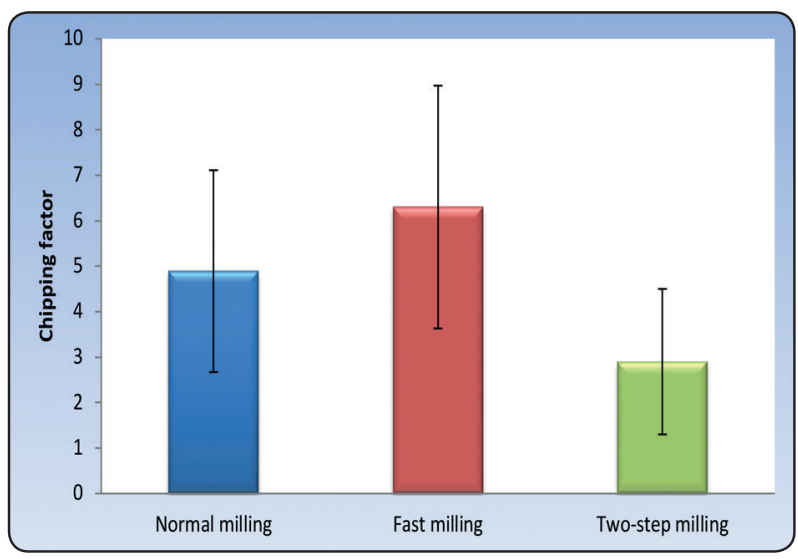

Fig. (3). Bar chart representing mean and standard deviation values for chipping factor values with different milling techniques regardless of ceramic type

\section{Interactions of variables: (Table 5, Fig 4)}

\section{Comparison between ceramic types}

With normal milling; there was a statistically significant difference between mean chipping factor values of ceramic types $(P$-value $<0.001$, Effect size $=0.930$ ). Pair-wise comparisons revealed that e.max showed the statistically significantly highest mean chipping factor value. There was no statistically significant difference between IPS Empress CAD and Vita Enamic; both showed the statistically significantly lowest mean chipping factor values.

With fast milling; there was a statistically significant difference between mean chipping factor values of ceramic types ( $P$-value $<0.001$, Effect size $=0.951)$. Pair-wise comparisons revealed that e.max showed the statistically significantly highest mean chipping factor value. IPS Empress CAD showed statistically significantly lower mean value. Vita Enamic showed the statistically significantly lowest mean chipping factor value.

With two-step milling; there was a statistically significant difference between mean chipping factor values of ceramic types $(P$-value $<0.001$, Effect size $=0.873)$. Pair-wise comparisons revealed that there was no statistically significant difference between IPS Empress CAD and e.max; both showed the statistically significantly highest mean chipping factor values. Vita Enamic showed the statistically significantly lowest mean chipping factor value.

\section{Comparison between milling techniques}

With IPS Empress CAD; there was a statistically significant difference between mean chipping factor values of milling techniques $(P$-value $<0.001$, Effect size $=0.581)$. Pair-wise comparisons revealed that fast milling showed the statistically significantly highest mean chipping factor value. Two-step milling showed statistically significantly lower mean value. Normal milling showed the statistically significantly lowest mean chipping factor value.

TABLE (5) The mean, standard deviation (SD) values and results of two-way ANOVA test for comparison between chipping factor values with different interactions of variables.

\begin{tabular}{|c|c|c|c|c|c|c|c|c|}
\hline \multirow{2}{*}{ Milling } & \multicolumn{2}{|c|}{ IPS Empress CAD } & \multicolumn{2}{|c|}{ Vita Enamic } & \multicolumn{2}{|c|}{ e.max } & \multirow{2}{*}{$\begin{array}{c}P \text {-value (Effect } \\
\text { of ceramic } \\
\text { type) }\end{array}$} & \multirow{2}{*}{$\begin{array}{c}\text { Effect size } \\
\text { (Partial Eta } \\
\text { Squared) }\end{array}$} \\
\hline & Mean & SD & Mean & SD & Mean & SD & & \\
\hline Normal milling & $3.2^{\mathrm{BF}}$ & 0.28 & $3.6^{\mathrm{BE}}$ & 0.34 & $7.86^{\mathrm{AE}}$ & 0.53 & $<0.001 *$ & 0.930 \\
\hline Fast milling & 4.87 BD & 0.6 & $4.15^{\mathrm{CD}}$ & 0.16 & $9.89 \mathrm{AD}$ & 0.35 & $<0.001 *$ & 0.951 \\
\hline Two-step milling & $3.96 \mathrm{AE}$ & 0.44 & $0.74^{\mathrm{BF}}$ & 0.07 & $3.99 \mathrm{AF}$ & 0.3 & $<0.001 *$ & 0.873 \\
\hline$P$-value (Effect of milling) & \multicolumn{2}{|c|}{$<0.001^{*}$} & \multicolumn{2}{|c|}{$<0.001 *$} & \multicolumn{2}{|c|}{$<0.001 *$} & & \\
\hline Effect size (Partial Eta Squared) & \multicolumn{2}{|c|}{0.581} & \multicolumn{2}{|c|}{0.869} & \multicolumn{2}{|c|}{0.947} & & \\
\hline
\end{tabular}

*: Significant at $P \leq \mathbf{0 . 0 5}$,

$A, B, C$ superscripts in the same row indicate statistically significant difference between ceramics

$D, E, F$ superscripts in the same column indicate statistically significant difference between milling techniques 


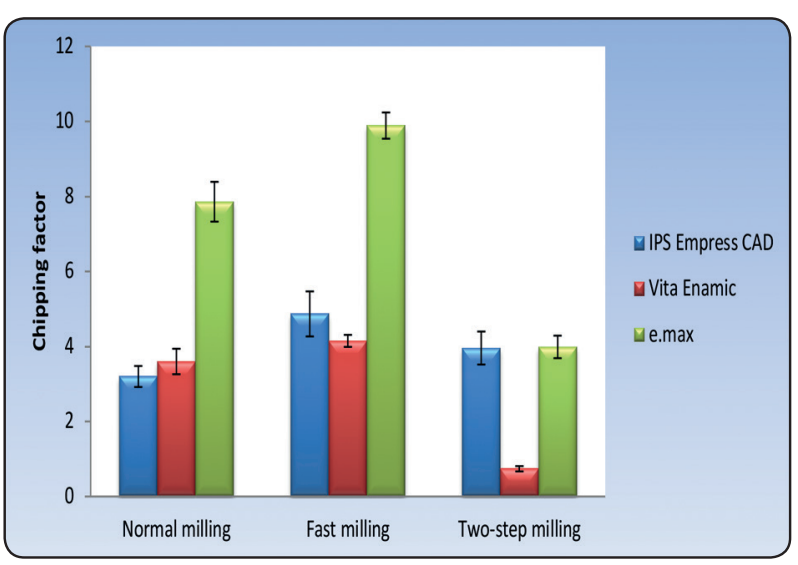

Fig. (4). Bar chart representing mean and standard deviation values for chipping factor values with different interactions of variables.

Whether with Vita Enamic or e.max; there was a statistically significant decrease in mean chipping factor values of milling techniques $(P$-value $<0.001$,
Effect size $=0.869)$ and $(P$-value $<0.001$, Effect size $=0.947)$, respectively. Pair-wise comparisons revealed that fast milling showed the statistically significantly highest mean chipping factor value. Normal milling showed statistically significantly lower mean value. Two-step milling showed the statistically significantly lowest mean chipping factor value.

As for the distribution of the peripheral marginal chippings of the different veneers as scored through the top view and stereomicroscopic images, figures 5-8 revealed that all the veneers showed cervical margin chippings $100 \%$, incisal margins particularly at the corners were nearly $22 \%$, while the axial peripheries showed $18 \%$ chippings.

Stereomicroscopic images (25X) showing the peripheral marginal chippings of the three different ceramic veneers milled with three different machining protocols.

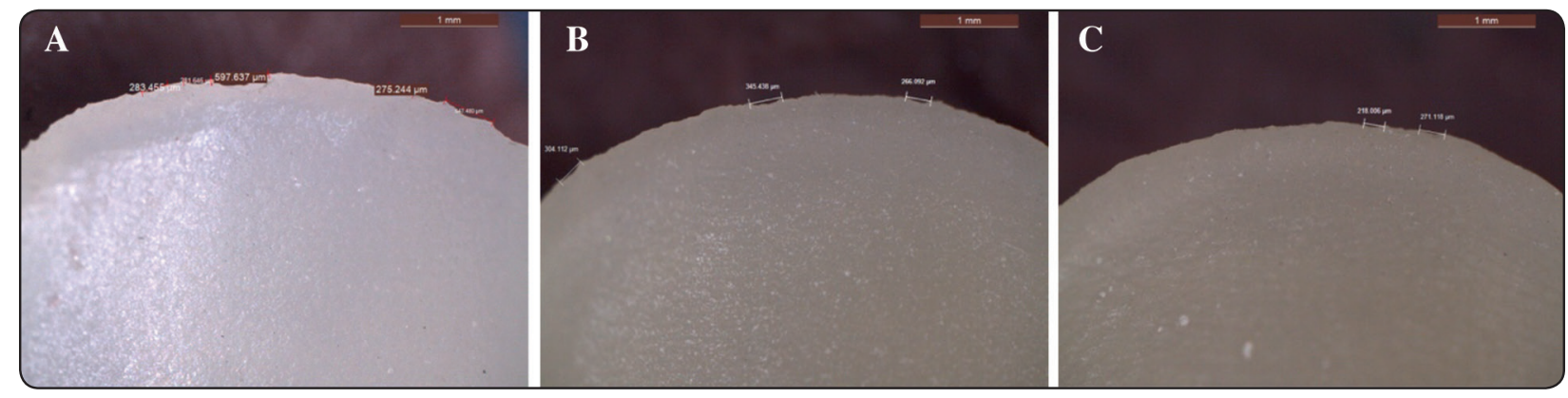

Fig (5) A: e.max/normal milling/ cervical margin. B: Empress CAD/normal milling/ cervical margin. C: Vita Enamic/normal milling/ cervical margin

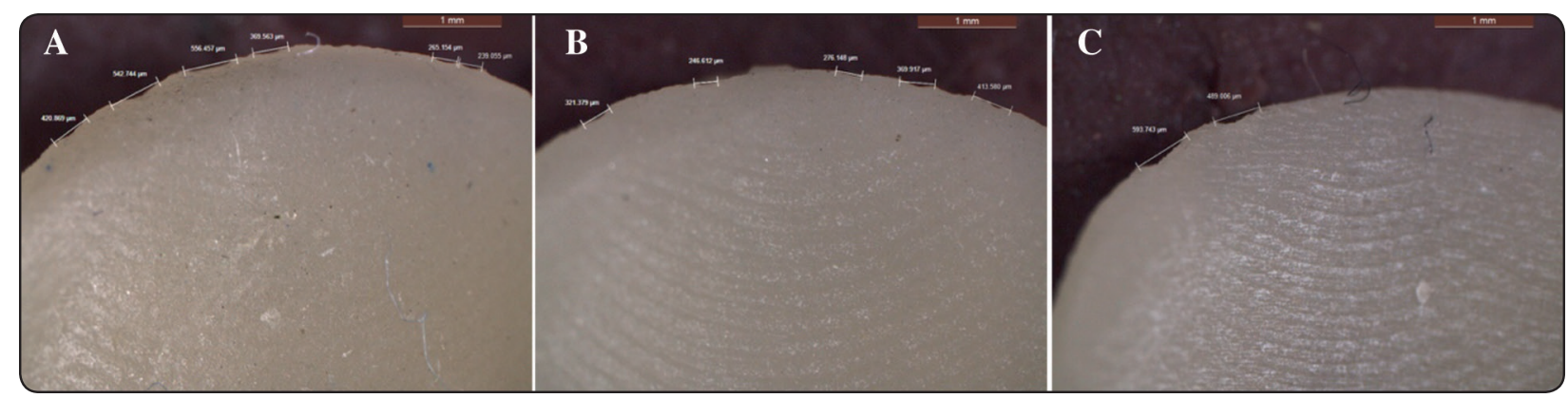

Fig (6) A: e.max/fast milling/ cervical margin. B Empress CAD/fast milling/ cervical margin. C: Vita Enamic/fast milling/ cervical margin 


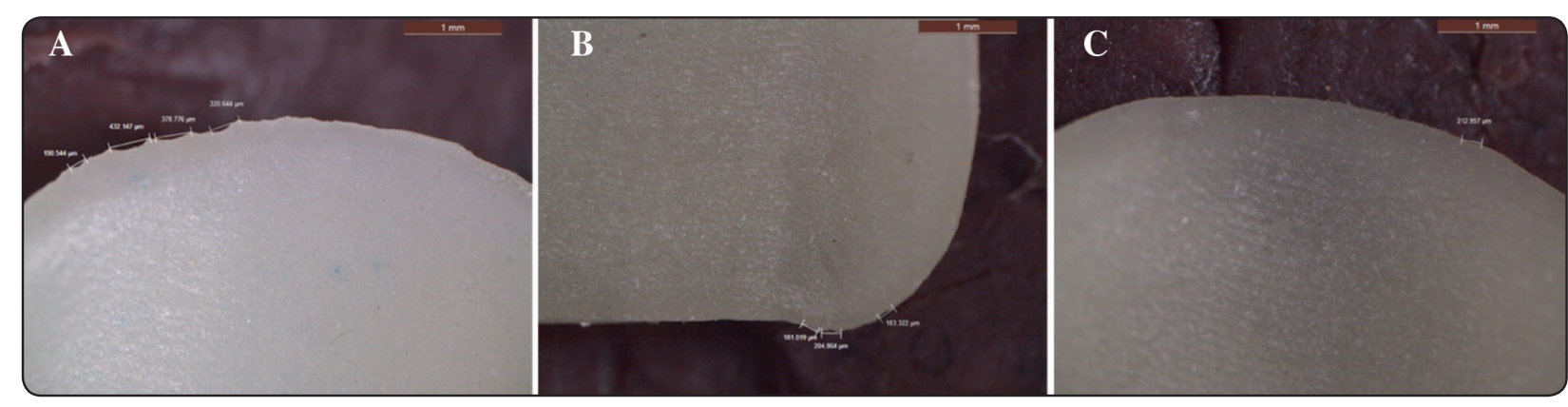

Fig. (7) A: e.max/2-step milling/ Cervical margin. B: Empress CAD/2-step milling/ incisal periphery. C: Vita Enamic/2-step milling/ cervical margin
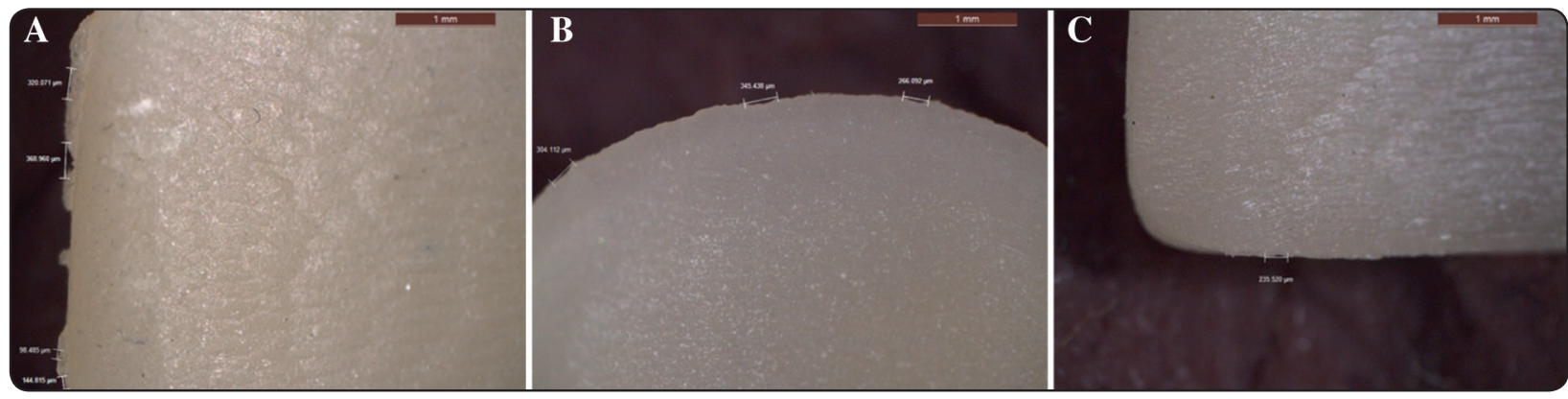

Fig. (8) A: e.max/fast milling/ axial periphery. B: Empress CAD/2-step milling/ cervical margin. C: Vita Enamic/fast milling/ incisal periphery

\section{DISCUSSION}

The degree of marginal fit of ceramic restorations cannot be considered the sole important factor for their successful performance, since the quality of their margins in terms of surface roughness and degree of smoothness together with being free of microscopic imperfections could also be a determining factor for their optimum success from the biologic and periodontal stand points. ${ }^{33-37}$

Along with the evidence based dental literature to support application of the minimally invasive ceramic restorations, ${ }^{2-4}$ together with the newly introduced ceramic and hybrid materials, it sounds logic to study the influence of different machining programs on the quality of the produced margins of the different veneer materials.

Therefore, the aim of the present investigation was to evaluate the effect of the machining speed and the CAD/CAM ceramic material on the edge chipping of three different labial ceramic veneers: lithium reinforced (LR), hybrid ceramic (HC), and lithium-disilicate (LD) in order to detect the behavior of materials with different mechanical properties on the marginal quality.

Other parameters like, for example, marginal or internal fit were not investigated in this study as the purpose of the present research was directed mainly to assess the peripheral marginal chipping of these different ceramic veneers.

Utmost care was undertaken to standardize the experimental design of this research in order to obtain accurate and realistic data. Therefore, production of the standard veneer ceramic samples was obtained from a single preparation of a maxillary central incisor through adopting the Cerec CAD/CAM technology. The measuring procedure 
as well, was achieved in such a meticulous manner following previous authors, ${ }^{18,24}$ through capturing of a digital top view image of the veneer margins to be transferred to the computer using a specific software analysis technology to measure the peripheral circumference of each veneer $(\mathrm{P})$.

The amount of peripheral marginal chippings (L) of each veneer was measured in microns using a stereomicroscope in order to calculate its chipping factor $(\mathrm{CF})$ using the formula $\mathrm{CF}=\mathrm{L} / \mathrm{Px} 100$, where $\mathrm{L}$ is the length of the chipped margins and $\mathrm{P}$ is the perimeter of the ceramic veneer.

According to the results of the present investigation the null hypothesis had to be rejected since both the ceramic type and the milling program revealed statistically significant effect on the mean chipping factor values of the different treatments (Table 2).

As regards the effect of ceramic type on the veneer marginal chipping regardless of the milling protocol (Table 3, Figure 2) there was a statistically significant difference between the mean chipping factor of the three tested ceramic types. The e.max CAD ceramic veneers showed the highest mean chipping factor value $(7.25 \pm 2.56)$, followed by IPS Empress CAD (4.01 \pm 0.83$)$, and the least mean $\mathrm{CF}$ value was revealed with Vita Enamic veneers. $(2.83 \pm 1.56)$. This finding might be in accordance with Quinn et al, ${ }^{(38)}$ who found that glass ceramic is more prone to marginal chipping than other materials. Tsitrou et al, ${ }^{(24)}$ found that the potential for marginal chippings increased as the brittleness index (BI) of a material increased. They reported also that the type of the milled restoration is a determining factor for the quality of the margins, particularly the minimally invasive procedures with CAD/CAM systems.

On the same line Porto et al, ${ }^{30}$ revealed that hard materials with high brittleness index perform more poorly than those with lower values as evidenced through their chipping susceptibility with CAD/CAM milling. Their results proved that lithium disilicate glass ceramic showed the highest BI values as well as Vickers hardness, and feldspar leucite reinforced glass ceramic showed intermediate values, whereas the nanofilled CAD/ CAM resin offered the lowest values. These results could be supportive and clarify the findings of our present investigation which revealed that the IPS e.max CAD (LD) showed the highest chipping factor mean values, followed by the IPS Empress CAD (LR), and the least CF mean values obtained with the Vita Enamic Hybrid ceramic (HC), where there was statistically significant difference between them.

These findings agree also with Tsitrou et al, ${ }^{24}$ who found that lithium disilicate glass ceramic (IPS e.max) showed the highest $\mathrm{CF}$ value and the leucite reinforced glass ceramic (Pro CAD) was next, while the composite material (Paradigm Mz 100) had the lowest $\mathrm{CF}$ value.

Giannetopoulos et al ${ }^{18}$ also realized that ceramic restorations with fine marginal finish line, as the case with minimally invasive procedures, are more subject to chipping during the milling process, which might also justify the findings of the present results.

Concerning the effect of the milling protocol regardless of the ceramic type (Table 4 and Figure 3 ), there was a statistically significant difference between the mean chipping factor of the three machining techniques. The fast milling showed the highest mean chipping factor value $(6.30 \pm 2.67)$, followed by normal milling $(4.89 \pm 2.22)$, and the least mean CF value was revealed with Two-Step milling $(2.90 \pm 1.60)$

Reich et al, ${ }^{10}$ reported that the marginal edges of ceramic restorations are stressed during manufacturing process, particularly CAD/CAM milling producing the characteristic chipping edge fractures, whereas the heat pressable types showed less prominent edge deficiencies. 
The results of the present study go along with Kirsch et al, ${ }^{39}$ who suggested that a specific milling strategy should be particularly recommended for glass ceramic restorations of the minimally invasive preparations. On this aspect also Bosch et al, ${ }^{40}$ found that five axis milling devices delivers high truness and good quality restorations than those milled with a 4-axial milling unit. However, they found that the 2-step mode was not significantly better than the 1-step mode, which contradicts our findings where the 2-step mode differed significantly from the normal, and fast milling modes as far as marginal chipping is concerned. This difference might be due to the main concern of their study which was restoration accuracy rather than marginal chipping.

Kirsch et al, ${ }^{39}$ and Bosch et al, ${ }^{40}$ reported that five axis machining is mostly used in labside fabrication. They also expected that labside machining would provide more accurate restorations than those produced through Chairside machining particularly for ceramic restorations with thin margins.

The results of the present study concerning the interaction of the tested variables (Table 5, Figure 4) regarding the comparison of three ceramic types with normal milling; Vita Enamic and IPS Empress CAD showed the least mean CF values which differed significantly from those revealed with e.max $\mathrm{CAD}$ which presented the highest mean $\mathrm{CF}$ values.

Fast milling protocol showed that e.max CAD presented the highest $\mathrm{CF}$ mean values followed by IPS Empress CAD and the least mean $\mathrm{CF}$ values were recorded for Vita Enamic.

As for the Two-step milling, Vita Enamic showed the lowest CF mean values, while IPS Empress $\mathrm{CAD}$ and e.max CAD presented higher mean $\mathrm{CF}$ values.

These results are in accordance with Porto et $\mathrm{al},{ }^{30}$ who found that hard ceramic materials revealed more chipping defects due to their high brittleness index (BI) which led to their poor performance with machinability compared to those with lower BI. Therefore, lithium disilicate (e.max CAD), in general, showed the highest $\mathrm{CF}$ mean values with the three machining speeds followed by leucite reinforced (IPS Empress CAD), and the least CF mean values were recorded with the hybrid ceramic Vita Enamic.

Despite these findings of some research papers, lithium disilicate glass ceramic is still one of the best esthetic materials as far as the clinical performance is concerned particularly if used correctly and properly handled. ${ }^{30,41-43}$

As for the comparison of the milling techniques in the different interaction of the tested variables (Table 5, Figure 4) the fast milling in general yielded the highest chipping factor (CF) mean values particularly with e.max CAD followed by the two-step and normal milling.

However, it has to be noticed that the hybrid ceramic Vita Enamic showed the best performance with the two-step milling followed by the normal milling and the least was revealed with the fast milling. The normal milling showed the best performance with IPS Empress CAD.

These statistically significant differences of the obtained data could be justified not only to the difference of the mechanical properties of the tested materials, particularly their hardness and BI, but also to the time elapsed during the machining process; where a fast milling might be hazardous with thin minimally invasive restorations. Therefore, the dentist while planning for treatment with these CAD/CAM esthetic restorations should select the optimum combination between the material and the milling strategy.

As for the interesting observation that was detected while scoring the number of chippings of the different veneer peripheries the cervical margins revealed the majority followed by the incisal and the lowest was for the axial periphery. This could be explained to the fact that curved cervical peripheries and inciso-axial angles are the most critical areas during milling rather than the straight axial peripheries. This might be in accordance with 
the findings of Kirsch et al, ${ }^{39}$ and Bosch et al, ${ }^{40}$ who revealed that steep walls and small angles could be machined properly using the Five-axis milling mainly adopted for labside machining rather than the Four-axis Chairside CAD/CAM devices.

However, Quinn et al, ${ }^{38}$ found that the Fouraxis (MCXL) extra-fine mode showed comparable results to those of the five-axis milling units.

\section{CONCLUSIONS}

From the results of the present in vitro investigation the following could be concluded:

1. The edge quality of thin ceramic CAD/CAM restorations proved to be material dependent, where harder materials like lithium di-silicate glass ceramic (IPS e.mac CAD) revealed significantly higher chipping factor $(\mathrm{CF})$ mean values than those of leucite reinforced ceramic (IPS Empress CAD) and Vita Enamic Hybrid Ceramic .

2. The machinability for producing thin $\mathrm{CAD} /$ CAM ceramic restorations influenced significantly their marginal quality as evidenced by the higher chipping factor mean values obtained with fast speed milling followed by the Normal and Two-step milling protocols.

3. The prosthodontist should be aware and knowledgeable about the mechanical properties of the recent $\mathrm{CAD} / \mathrm{CAM}$ ceramic materials in order to choose the optimum one with the convenient milling protocol during the treatment planning to get the best clinical performance of these highly esthetic restorations.

\section{RECOMMENDATIONS}

- The prevalence of this chipping phenomena particularly with thin ceramic margins necessitates finishing and polishing of these margins under magnification for the sake of producing smooth margins that would be the optimum from the biologic point of view.
- Selection of the suitable ceramic material according to the esthetic and functional demands that are needed in the restorative site has to be of prime concern for the prosthodontist.

- The selected machining program should be the one that is most convenient with the chosen ceramic material.

\section{SUGGESTIONS FOR FURTHER INVESTIGATION}

- Since minimally invasive restorations are becoming a modern trend for the sake of conservatism and ultrathin veneers are strongly recommended, therefore, it is worthy to investigate the influence of the machinability on the edge chipping of these veneers with different thicknesses.

- Other machining systems also for example Five-axis-milling strategy would be another experimental solution that needs to be investigated which might deliver better margin quality with these types of thin ceramic restorations which are very technique sensitive.

\section{REFERENCES}

1. Fasbinder DJ. Clinical performance of chairside CAD/ CAM restorations. J. Amer Dent Assoc, 2006; 137 (suppl.): 225-31 S.

2. Tsitrou EA, van Noort R. Minimal preparation designs for single posterior indirect prostheses with the use of Cerec system. Int. J. Computerised Dent 2008; 11: 227-40.

3. Reich S, Petschelt A, Lohbauer U. The effect of finish line preparation and layer thickness on the failure load and fractography of Zr O2 Copings.J. Prosthet Dent 2008; 99: 369-76.

4. Tsitrou EA, Northeast SE, van Noort R. Evaluation of the marginal fit of three margin designs of resin composite crowns using CAD/CAM. J. Dentistry 2007; 35: 68 - 73.

5. Guess PC, Vagkopoulou T, Zhang Y, Wolkewitz M, Strub JR. Marginal and internal fit of heat pressed versus CAD/ CAM fabricated all-ceramic onlays after exposure to thermo-mechanical fatigue. J Dentistry 2014; 42 (2): 199 - 209.

6. Magne P, Schlichting LH, Maia HP, Baratieri LN. In vitro fatigue resistance of $\mathrm{CAD} / \mathrm{CAM}$ composite resin and ceramic posterior occlusal veneers. J Prosthet Dent. 2010; 104 (3): 149 - 57. 
7. Andrade JP, Stana D, Bittencourt HR, Borges GA, Burnett LHJ, Spohr AM. Effect of different computer-aided design/computer aided manufacturing (CAD/CAM) materials and thicknesses on the fracture resistance of occlusal veneers. Oper Dent. 2018; 43 (5): 439 - 48.

8. Schmidt K, Chiayabutr Y, Phillips K, Kois J. Influence of preparation design and existing condition of tooth structure on load to failure of ceramic laminate veneers. J Prosthet Dent. 2011; 105: 374 - 382.

9. Strassler HE, Minimally invasive porcelain veneers: indication for a conservative esthetic dentistry treatment modality. Gen Dent. 2007; 55 (7): 686 - 94.

10. Reich S, Gozdowski S, Trentzsch L, Frankenberger R, Lohbauer U. Marginal fit of heat-pressed VS CAD/CAM Processed all-ceramic onlays using a milling unit prototype. Oper. Dent 2008; 33 - 6, 644 - 650.

11. Reiss B. Clinical results of cerec inlays in a dental practice over a period of 18 years. Inter. J. of Comput. Dent. 2006; 9 (1) $11-22$.

12. Lohbauer U, Kramer N, Petschelt A, Frankenberger R. Correlation of in vitro fatigue data and in vivo clinical performance of a glass ceramic material. Dent. Mater 2008; 24 (1): 39 - 44.

13. Kramer N, Lohbauer U, Frankenberger R. Adhesive luling of indirect restorations. Amer J. of Dentistry 2000; 13: 60 D - 76 D.

14. Manhart J, Chen H, Hamm G, Hickel R. Buonocore memorial lecture. Review of the clinical survival of direct and indirect restorations in posterior teeth of the permanent dentition. Oper Dent 2004; 29 (5): 481 - 508.

15. Beschnidt SM, Strub JR. Evaluation of the marginal accuracy of different all-ceramic crown systems after simulation in the artificial mouth. J O Rehab 1999; 26: 582 - 593.

16. Goldman M, Leosonthorn P, White RR. Microleakage - full crowns and the dental pulp. J Endodontics 1982; 18: 473 .

17. Saridag S, Sevimay M, Pekkan G. Fracture resistance of teeth restored with all-ceramic inlays and onlays: an in vitro study. Oper Dent. 2013; 38 (6): 626-34.

18. Giannetopoulos S, van Noort R, Tsitrou E. Evaluation of the marginal integrity of ceramic copings with different marginal angles using two different CAD/CAM systems. J Dent. 2010; 38 (12): 980-6.
19. Guess PC, Schultheis S, Wolkewitz M, Zhang Y, Strub JR. Influence of preparation design and ceramic thicknesses on fracture resistance and failure modes of premolar partial coverage restorations. J Prosthet Dent. 2013; (4): 264 - 73.

20. Magne P, Cheung R. Numeric simulation of occlusal interferences in molars restored with ultrathin occlusal veneers. J Prosthet Dent. 2017; 117 (1): 132 - 7.

21. Rekow ED. High-technology innovations and limitations for restorative dentistry. Dental Clinics of North America. 1993; 37: 513 - 24.

22. Shearer AC, Heymann HO, Wilson NH. Two ceramic materials compared for the production of CEREC inlays. J Dentistry 1993; 21: 302 - 4 .

23. Sindel J, Petschelt A, Grellner F, Dierken C, Greil P. Evaluation of subsurface damage in CAD/CAM machined dental ceramics. J. of Materials Science - Materials in Medicine 1998; 9: 291 - 5 .

24. Tsitrou EA, Northeast SE, van Noort R. Brittleness index of machinable dental materials and its relation to the marginal chipping factor. J. Dentistry 2007; 35 (12): 897 - 902.

25. Baik DS, No KS, Chun JS, Yoon YJ, Cho HY. A comparative evaluation method of machinability formica-based glass ceramics. J of Materials Science 1995; 30: 1801-6.

26. Taira M, Yamaki M. Ranking machinability of 9 machinable ceramics by dental high-speed cutting tests. $\mathrm{J}$ of Materials Science Letters 1994; 13: 480 - 2.

27. Boccaccini AR. Machinability and brittleness of glassceramics. J of Materials Processing Technology 1997; 65: 302-4.

28. Sihgal J, Nakao Y, Takahashi H, Ito S. Brittleness of glasses by indentation. J. of Materials Science Letters 1995; 14: $167-9$.

29. Boccaccini AR. Assessment of brittleness of glass-ceramics and particulate glass matrix composites by indentation data. J. of Materials Science Letters 1996; 15: 1119 - 1121.

30. Porto TS, Roperto RC, Teich ST, Faddoul FF, Rizzante FA, Porto-Neto ST, Campos EA. Brittleness index and its relationship with materials mechanical properties: Influence on the machinability of CAD/CAM materials. Braz. Oral Res. 2019; 33: e26.

31. Chavali R, Nejat AH, Lawson NC. Machinability of CADCAM materials. J Prosthet Dent 2017 Aug; 118 (2):194-9. 
32. Carl Hany and Maha Tymour. Marginal integrity of glass ceramic laminate veneers produced through different CAD/CAM milling protocols: In-vitro Study. Egypt. Dent. J 2019; July 65: 1343 - 1354 .

33. Contrepois M, Soenen A, Bartala M, Laviole O. Marginal adaptation of ceramic crowns: a systematic review. J Prosthet Dent 2013, 110: 447 - 454.

34. Kosifaki P, del Pilar Pinilla Martin M, Strub JR. Relationship between crowns and the periodontium: a literature update. Quintessence Int 2010, 41: 109 - 126.

35. Yuksel E, Zaimoglu A. Influence of marginal and cement types on microleakage of all-ceramic crown systems. Braz oral Res 2011, 25: 261 - 266.

36. Baig MR, Tan KB, Nicholls JI. Evaluation of the marginal fit of zirconia ceramic computer-aided machined (CAM) crown system. J Prosthet Dent 2010, 104: 216 - 227.

37. Sax C, Hämmerle CH, Sailer I. 10-year clinical outcomes of fixed dental prostheses with zirconia frameworks. Int Comput Dent 2011, 14: 183 - 202.

38. Quinn GD, Giuseppetti AA, Hoffman KH. Chipping fracture resistance of dental CAD/CAM restorative materials: Part 1 procedures and results. Dent Mater 2014; 30:99-111.

39. Kirsch C, Ender A, Attin T, Mehl A. Truness of four milling procedures used in dental CAD/CAM systems. Clinical Oral Invest 2017; 21: 551 - 558.

40. Bosch G, Ender A, Mehl A: A 3-dimensional accuracy analysis of chairside CAD/CAM milling processes. J Prosthet Dent 2014; 112: 1425 - 1431.

41. Sener-Yamaner ID, Sertgöz A, Toz-Akalin T, Özcan M. Effect of material and fabrication technique on marginal fit and fracture resistance of adhesively luted inlays made of CAD/CAM ceramics and hybrid materials. J Adhes Sci Technol: 2017; 31 (1): 55 - 70.

42. Song XF, Ron HT, Yin L. Machinability of lithium disilicate glass ceramic in in vitro dental diamond bur adjusting process. J Mech Behav Biomed Mater. 2016 Jan; 53:78-92.

43. Leung BT, Tsoi JK, Matinlinna JP, Pow EH. Comparison of mechanical properties of three machinable ceramics with an experimental Fluorophlogopite glass ceramic. J Prosthet Dent 2015; 114: 440 - 446. 